\title{
Analysis of oral microbiota in patients with obstructive sleep apnea-associated hypertension
}

\author{
Chih-Yuan Ko ${ }^{1,2,3,4} \cdot$ An-Ke Hu $u^{1,2,3} \cdot$ Dylan Chou ${ }^{5} \cdot$ Li-Mei Huang ${ }^{1,2,3} \cdot$ Huan-Zhang Su, ${ }^{1,2,3} \cdot$ Fu-Rong Yan ${ }^{1,2,3,6}$. \\ Xiao-Bin Zhang ${ }^{1,2,3} \cdot$ Hua-Ping Zhang ${ }^{1,2,3} \cdot$ Yi-Ming Zeng ${ }^{1,2,3}$
}

Received: 20 December 2018 / Revised: 28 February 2019 / Accepted: 9 March 2019 / Published online: 11 April 2019

(c) The Author(s) 2019. This article is published with open access

\begin{abstract}
Obstructive sleep apnea-hypopnea syndrome (OSAHS) is an independent risk factor for hypertension (HTN). The oral microbiota plays a pathophysiological role in cardiovascular diseases; however, there are few reports directly investigating and identifying the organisms involved in OSAHS-related HTN. Therefore, this study aimed to identify those organisms. We obtained 139 oral samples and determined the microbiome composition using pyrosequencing and bioinformatic analyses of the 16S rRNA. We examined the fasting levels of cytokines and homocysteine in all participants and analyzed the correlations between the oral microbiota and homocysteine levels. We determined the molecular mechanism underlying HTN by investigating the genetic composition of the strains in the blood. We detected higher relative abundances of Porphyromonas and Aggregatibacter and elevated proinflammatory cytokines in patients with OSAHS of varying severity compared with individuals without OSAHS; however, the two organisms were not measured in the blood samples from all participants. High levels of specific Porphyromonas bacteria were detected in patients with OSAHS with and without HTN, whereas the relative abundance of Aggregatibacter was negatively correlated with the homocysteine level. The receiver operating characteristic curve analysis of controls and patients with OSAHS resulted in area under the curve values of 0.759 and 0.641 for patients with OSAHS with or without HTN, respectively. We found that the predictive function of oral microbiota was different in patients with OSAHS with and without HTN. However, there was no direct invasion by the two organisms causing endothelial cell injury, leading to speculation regarding the other mechanisms that may lead to HTN. Elucidating the differences in the oral microbiome will help us understand the pathogenesis of OSAHS-related HTN.
\end{abstract}

Keywords homocysteine $\cdot$ hypertension $\cdot$ oral microbiota $\cdot$ obstructive sleep apnea-hypopnea syndrome $\cdot$ periodontopathic bacteria

These authors contributed equally: Chih-Yuan Ko, An-Ke Hu, Dylan Chou

These authors jointly supervised this work: Chih-Yuan Ko, Hua-Ping Zhang, Ming Zeng

Supplementary information The online version of this article (https:// doi.org/10.1038/s41440-019-0260-4) contains supplementary material, which is available to authorized users.

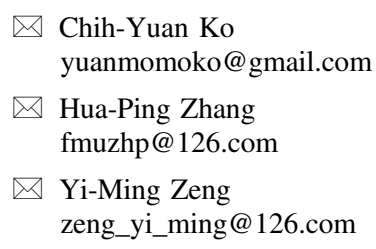

Extended author information available on the last page of the article.

\section{Introduction}

Recent evidence has shown an association between obstructive sleep apnea-hypopnea syndrome (OSAHS), a common sleep breathing disorder, and increased cerebral/ cardiovascular morbidity and mortality [1]. Hypertension (HTN) is a systemic disorder and the most prevalent cardiovascular disease (CVD) [2]. Its prevalence among primary and antihypertensive agent-resistant patients with OSAHS is $~ 65-80 \%$ [3, 4], and because blood pressure values also have a significantly positive relationship with the apnea-hypopnea index (AHI), the effects of OSAHS will continue to grow with increasing severity of OSAHS [5]. Hence, more reliable biomarkers and noninvasive screening techniques are needed.

Numerous surveys have implicated microbiota in the pathogenesis of HTN [6-10]. A delicate balance in the 
microbiota composition is fundamental for maintaining immunity and whole-body homeostasis [11]. Ideally, symbiotic bacteria maintain a constant relationship with the host, which can be compromised by changes in the microbiota composition, termed dysbiosis [12], thereby mediating immune dysregulation [13].

Interestingly, the characteristics of oral bacteria can reflect systemic disorders, including CVDs, coronary heart disease, and HTN $[6,8,10]$. HTN correlates positively with periodontopathic bacterial infections, particularly those caused by Aggregatibacter spp. and Porphyromonas spp [6, 14]. Furthermore, compared with healthy individuals, patients with OSAHS have considerably higher oral bacterial counts and an increased incidence of periodontitis, including those involving the aforementioned organisms [15]. OSAHS is also characterized by increasing levels of proinflammatory cytokines in the saliva and serum [15], which mediate endothelial cell injury and promote the occurrence and development of HTN [16].

However, the precise effect of oral microbiota on HTN in OSAHS has not yet been clarified. Currently, the entry of periodontal microorganisms and their metabolic products into systemic circulation is regarded as one mechanism that initiates HTN $[17,18]$. Therefore, oral bacterial flora is an important factor warranting further study with respect to its role in the development of HTN in OSAHS.

The present study aimed to determine the possible underlying mechanism by evaluating the relationship between oral microbiota and HTN in patients with OSAHS and examining whether the serum of patients with OSAHS contains DNA from Aggregatibacter spp. and Porphyromonas spp. Furthermore, homocysteine (HCY) levels are associated with the risk of CVD and HTN in OSAHS; $[19,20]$ therefore, we also assessed the correlation between the oral microbiota and HCY levels.

\section{Materials and methods}

\section{Subjects}

In total, 139 subjects were recruited and underwent a full night (from 2200 to 0800 hours) of polysomnography (PSG; SOMNOscreen ${ }^{\mathrm{TM}}$ plus $\left(\mathrm{PSG}^{+}\right)$, SOMNOmedics GmbH, Randersacker, Germany) conducted by technologists in a sleep laboratory at the Department of Respiratory and Critical Care Medicine. Fasting blood and oral mucosal (involving buccal mucosa, tongue, soft palate, and hard palate) samples were collected the following morning. The present study was approved by the Second Affiliated Hospital of Fujian Medical University's Institutional Review Board with the certificate number IRB No. 2017-78.

\section{OSAHS assessments}

PSG was performed with a computerized polysomnographic system, which simultaneously recorded electrocardiography, electroencephalography, electromyography, electrooculography, and dynamic blood pressure measurements, including systolic blood pressure (SBP) and diastolic blood pressure (DBP). A high blood pressure was defined as $\mathrm{SBP} \geq 140 \mathrm{mmHg}$ and/or DBP $\geq 90 \mathrm{mmHg}$ or self-reported use of medications for HTN [4]. Following the examination, AHI was calculated as the total number of episodes of apnea (continuous cessation of airflow for at least $10 \mathrm{~s}$ ) and hypopnea (reduction in airflow for $\geq 10$ s with oxygen desaturation of $\geq 4 \%$ ) by dividing the total sleep period by the number of episodes, according to the diagnostic criteria of the American Academy of Sleep Medicine. Adults with AHI $\leq 5$ were used as controls; thus, $5<$ AHI $\leq 15$ was considered to indicate mild OSAHS and AHI $\geq 15$ was considered to indicate moderate-to-severe OSAHS [5].

\section{Examination of $\mathrm{HCY}$}

The fasting HCY levels were examined with the Automatic Biochemical Analyzer (TBA-120 FR, Toshiba, Japan) and HCY assay kit (Yong He Sun Biotech. Ltd., Hunan, China), using the enzymatic cycling method.

\section{Cytokine analysis}

Interleukin (IL)-2, IL-4, IL-6, IL-10, tumor necrosis factor alpha, and interferon-gamma (IFN- $\gamma$ ) were assayed with the BD $^{\text {TM }}$ Cytometric Bead Array Kit Human Enhanced Sensitivity Flex Set (BD Biosciences, New Jersey, USA), as described previously [21]. The standard coefficient of determination was $>0.995$.

\section{Sampling, DNA extraction, and 16S rRNA gene amplification sequencing}

Once collected, all fresh oral mucosal samples were stored in a Microbiome Test Kit (G-BIO Biotech, Inc., Hangzhou, China). Magnetic bead isolation was used to extract the genomic DNA with a TIANamp Stool DNA Kit (TIANGEN Biotech (Beijing) Co., Ltd., China), according to the instructions. The NanoDrop ${ }^{\mathrm{TM}}$ ND-1000 Spectrophotometer (Thermo Fisher Scientific, Massachusetts, USA) was used to determine the concentration of the extracted DNA, and $1.0 \%$ agarose gel electrophoresis with $0.5 \mathrm{mg} / \mathrm{mL}$ ethidium bromide was used to confirm the quality.

Isolated fecal DNA was used as a template for polymerase chain reaction amplification (forward primer, 5'ACTCCTACGGGAGGCAGCAG-3'; reverse primer, 5'GGACTACHVGGGTWTCTAAT-3') of the V3 and V4 
hypervariable regions of the bacterial $16 \mathrm{~S}$ ribosomal RNA gene. The $16 \mathrm{~S}$ target-specific sequence contained adaptor sequences, allowing uniform amplification of a highly complex library for downstream next-generation sequencing on the Illumina MiSeq system (Illumina ${ }^{\circ}$ Inc., California, USA). Negative DNA extraction controls (lysis buffer and kit reagents only) were amplified and sequenced as contamination controls. The amplicons were normalized, pooled, and sequenced on the Illumina ${ }^{\circ}$ MiSeq platform using a V3 reagent kit with $2 \times 300$ cycles per sample and the prepared, imported routine data (samsheet) run in the MiSeq sequence program. After sequencing, the Q30 scores were $\geq 70 \%$, the cluster passing filter (cluster PF) was $\geq 80 \%$, and there were at least 30,000 clean tags. Finally, image analysis and base calling were performed using the MiSeq Control Software.

\section{Porphyromonas gingivalis and Aggregatibacter actinomycetemcomitans measurement in the blood}

A magnetic bead isolation kit (Magen, Guangzhou, China) was used to extract genomic DNA from all blood samples, according to the instructions. Using real-time PCR, these samples were then examined for DNA from two different bacterial species: $P$. gingivalis (forward primer, 5'-TGTAGATGACTGATGGTGAAAACC-3'; reverse primer 3'-A CGTCATCCACACCTTCCTC-5') and A. actinomycetemcomitans (forward primer 5'-CTTACCTACTCTTGAC ATCCGAA-3'; reverse primer 3'-ATGCAGCACCTGT CTCAAAGC- $5^{\prime}$ ). The real-time PCR protocol comprised an initial step of predenaturation at $95^{\circ} \mathrm{C}$ for $10 \mathrm{~min}$, followed by 40 denaturation cycles at $95^{\circ} \mathrm{C}$ for $15 \mathrm{~s}$ and annealing at $61.5^{\circ} \mathrm{C}$ for $30 \mathrm{~s}$.

\section{Bioinformatic, predictive function, and statistical analyses}

The total sequence data were used to analyze the taxa of the oral mucosal microbiota based on the Quantitative Insights into Microbial Ecology bioinformatic pipeline for performing taxonomy assignments using the operational taxonomic unit method. The Phylogenetic Investigation of Communities by Reconstruction of Unobserved States bioinformatics software package and Kyoto Encyclopedia of Genes and Genomes were used to predict the bacterial metabolic functions.

Differences in oral microbiota were analyzed by the Kruskal-Wallis test, as appropriate. Principal coordinate analysis, based on the Bray-Curtis dissimilarity and $\mathrm{R}$ statistics, was also performed. Further data analysis by $t$-test or one-way analysis of variance (ANOVA) was undertaken with SPSS version 19.0 (SPSS Inc., Chicago, IL, USA), and the data are expressed as the mean \pm standard deviation
(SD). Significant differences within groups were analyzed with ANOVA, followed by post hoc Fisher's least significant difference corrections for multiple comparisons of normally distributed variance data. We considered a twosided $p$ value $<0.05$ to be statistically significant. The correlation coefficients between oral microbiota and HCY level were evaluated by Spearman's correlation.

\section{Results}

\section{Participants' characteristics}

We enrolled 126 patients with OSAHS (mild $(n=35)$ and moderate-to-severe $(n=91)$ ) and 13 controls (Table 1$)$. The age of those with moderate-to-severe OSAHS was significantly higher than that of the controls, and body weight was the highest in the moderate-to-severe OSAHS group. Conversely, the body mass index (BMI) and waist circumferences of the controls were the lowest (Table 1).

The AHI, hypopnea index, and mean $\mathrm{SpO}_{2}$ were the highest in patients with moderate-to-severe OSAHS, whereas the lowest $\mathrm{SpO}_{2}$ was in those with mild OSAHS (Table 1). Both the average SBP and DBP were the highest in patients with moderate-to-severe OSAHS (Table 1). We also examined patients with OSAHS and HTN, dividing them into five groups (see supplementary Table 1 for the detailed data).

\section{Changes in taxa among groups}

Simpson's diversity index detected no statistically significant differences in community richness and diversity among the control group and other groups (see supplementary Figure 1 for details).

The relative abundances of the Porphyromonas and Aggregatibacter genera in Group 1 were significantly higher than those in the controls. Treponema, Abiotrophia, Hydrotalea, and Schlegelella were also significantly more abundant in Group 2 than in the controls. However, Kingella, Fusicatenibacter, Mobiluncus, $f$ _Clostridiaceae 1, Fluviicola, Clostridium III, $p$ __Actinobacteria, o__Acidimicrobiales, Parcubacteria_genera_incertae_sedis, $f$ _Leptotrichiaceae, Lysobacter, Pirellula, f_Verrucomicrobiaceae, Rheinheimera, $f$ _Geodermatophilaceae, Methylobacillus, Anaerococcus, c_Acidobacteria_Gp4, Aciditerrimonas, f_Parachlamydiaceae, Ulvibacter, Gp21, Gp7, and Latescibacteria_genera_incertae_sedis genera were more abundant in the controls than in those with OSAHS (Fig. 1a; see supplementary Table 2 for detailed statistics).

The relative abundance of the Porphyromonas genera was significantly higher in Group 1 than in the controls, as were the relative abundances of Abiotrophia in Group 2 and Group 4 
Table 1 Participant characteristics

\begin{tabular}{|c|c|c|c|c|c|c|c|c|}
\hline & \multirow{3}{*}{$\begin{array}{l}\text { Control } \\
(n=13)\end{array}$} & \multirow{3}{*}{$\begin{array}{l}\text { Group1 } \\
(n=35)\end{array}$} & \multirow{3}{*}{$\begin{array}{l}\text { Group2 } \\
(n=91)\end{array}$} & \multirow[t]{3}{*}{$\mathrm{F}$ value } & \multirow[t]{3}{*}{$P$ value } & \multicolumn{3}{|c|}{ post-hoc test } \\
\hline & & & & & & \multicolumn{3}{|l|}{$P$ value } \\
\hline & & & & & & C vs G1 & $\mathrm{C}$ vs G2 & G1 vs G2 \\
\hline Gender (male/female) & $9 / 4$ & $31 / 4$ & $81 / 10$ & $\mathrm{NA}^{\mathrm{a}}$ & NA & NA & NA & NA \\
\hline Age (years, mean $\pm \mathrm{SD}$ ) & $35.92 \pm 7.69$ & $43.57 \pm 12.13$ & $46.66 \pm 13.35$ & 4.352 & 0.015 & 0.065 & 0.005 & 0.221 \\
\hline Height $(\mathrm{cm})$ & $167.92 \pm 9.07$ & $167.44 \pm 7.48$ & $167.42 \pm 7.27$ & 0.026 & 0.975 & NA & NA & NA \\
\hline Weight $(\mathrm{kg})$ & $68.40 \pm 9.28$ & $72.87 \pm 11.29$ & $78.67 \pm 13.53$ & 5.398 & 0.006 & 0.279 & 0.007 & 0.023 \\
\hline Body mass index $\left(\mathrm{kg} \mathrm{m}^{-2}\right)$ & $24.10 \pm 2.33$ & $26.75 \pm 4.79$ & $27.68 \pm 3.61$ & 5.118 & 0.007 & 0.036 & 0.002 & 0.227 \\
\hline Waist circumference $(\mathrm{cm})$ & $83.85 \pm 6.26$ & $93.51 \pm 11.27$ & $96.19 \pm 8.33$ & 10.884 & $<0.001$ & 0.001 & $<0.001$ & 0.137 \\
\hline Hip circumference $(\mathrm{cm})$ & $97.81 \pm 4.10$ & $99.00 \pm 7.77$ & $100.54 \pm 6.15$ & 1.471 & 0.233 & NA & NA & NA \\
\hline Homocysteine (umol/L) & $11.29 \pm 3.13$ & $15.43 \pm 6.67$ & $16.27 \pm 8.07$ & 2.572 & 0.080 & NA & NA & NA \\
\hline Sleep efficiency $(\%)$ & $68.80 \pm 17.65$ & $68.74 \pm 17.92$ & $72.08 \pm 15.17$ & 0.668 & 0.515 & NA & NA & NA \\
\hline Arousal index (events/h) & $3.57 \pm 1.87$ & $3.35 \pm 1.71$ & $3.23 \pm 2.44$ & 0.148 & 0.863 & NA & NA & NA \\
\hline Apnea-hypopnea index (events/h) & $1.83 \pm 1.34$ & $9.19 \pm 3.13$ & $46.44 \pm 21.66$ & 78.109 & $<0.001$ & 0.202 & $<0.001$ & $<0.001$ \\
\hline Hypopnea index (events/h) & $1.21 \pm 0.91$ & $6.27 \pm 3.19$ & $17.16 \pm 11.19$ & 29.050 & $<0.001$ & 0.094 & $<0.001$ & $<0.001$ \\
\hline Mean $\mathrm{SpO}_{2}(\%)$ & $95.62 \pm 1.12$ & $94.86 \pm 1.46$ & $92.81 \pm 2.42$ & 18.299 & $<0.001$ & 0.273 & $<0.001$ & $<0.001$ \\
\hline Lowest $\mathrm{SpO}_{2}(\%)$ & $90.62 \pm 3.28$ & $85.11 \pm 4.71$ & $75.30 \pm 8.79$ & 37.327 & $<0.001$ & 0.027 & $<0.001$ & $<0.001$ \\
\hline Average systolic blood pressure $(\mathrm{mmHg})$ & $109.15 \pm 19.94$ & $112.94 \pm 32.43$ & $128.76 \pm 25.36$ & 6.253 & 0.003 & 0.665 & 0.015 & 0.004 \\
\hline Average diastolic blood pressure $(\mathrm{mmHg})$ & $74.31 \pm 11.43$ & $77.83 \pm 22.05$ & $86.31 \pm 14.80$ & 13.28 & $<0.001$ & 0.116 & 0.001 & 0.024 \\
\hline
\end{tabular}

${ }^{a}$ N/A: not analyzed. Control: apnea-hypopnea index (AHI) $\leq 5$ (non-OSAHS), Group 1: $5<\mathrm{AHI} \leq 15$ (mild OSAHS with/without hypertension), Group 2: AHI > 15 (moderate-to-severe OSAHS with/without hypertension); the statistical analysis was performed with Fisher's least significant difference test

and Hydrotalea and Kingella in Group 4. Gemmiger was significantly more abundant in Group 2 than in Group 1. Gammaproteobacteria_incertae_sedis and Syntrophomonas were significantly more abundant in Group 3 than in the controls. However, Schlegelella, $f$ _Clostridiaceae 1, Fluviicola, Clostridium III, $p \_$_Actinobacteria, o__Acidimicrobiales, Parcubacteria_genera_incertae_sedis, Lysobacter, Pirellula, f_Verrucomicrobiaceae, Rheinheimera, f_Geodermatophilaceae, Methylobacillus, Anaerococcus, c_Acidobacteria_Gp4, Aciditerrimonas, $f$ _Parachlamydiaceae, Ulvibacter, Gp21, Gp7, Latescibacteria_genera_incertae_sedis, and Janthinobacterium genera were more abundant in the controls than in those with OSAHS (Fig. 2a; see supplementary Table 3 for detailed statistics).

\section{Cytokine analysis}

The levels of IL-2 $[\mathrm{F}(2,138)=3.211, p=0.043]$ and IFN- $\gamma$ $[\mathrm{F}(2,138)=3.987, \quad p=0.021]$ were significantly higher among patients with OSAHS than among the controls; post hoc testing indicated higher levels among those with AHI $\geq 15$ than among those with AHI $<15$ (Fig. 1b).

\section{Predictive function analysis}

The relative abundances of energy and biotin metabolism were significantly higher in Group 1 than in the controls, whereas methane and thiamin metabolism were significantly higher in Group 1 than in the controls and Group
2. Protein folding and associated processing were significantly higher in Group 3 than in Group 2. The relative abundances of cell motility and secretion, protein kinases, cyanoamino acid metabolism, and ion channels were significantly higher in Group 4 than in Group 1. Conversely, mitogen-activated protein kinase signaling pathway-yeast was significantly higher in Group 1 than in Group 4, and porphyrin and chlorophyll metabolism was significantly higher in Group 1 than in both Group 4 and Group 2. Bacterial invasion of epithelial cells was significantly higher in Group 2 than in the controls (Fig. 2b; detailed statistics are presented in supplementary Table 4).

\section{Oral microbiota discrimination predictive model}

To select predictive features of oral microbiomes, we discriminated between patients with OSAHS and those with OSAHS without HTN and controls with areas under the receiver operating characteristic curve (ROC-AUCs) of 0.759 and 0.750 , respectively, and discriminated patients with OSAHS from those with OSAHS without HTN with an ROC-AUC of 0.641 (Fig. 2c).

\section{Quantitative detection of $18 \mathrm{~S}$ rDNA genes of $P$. gingivalis and A. actinomycetemcomitans in serum}

Neither $P$. gingivalis nor $A$. actinomycetemcomitans $18 \mathrm{~S}$ recombinant DNA genes were detected in the 139 samples (see supplementary Figure 2 for details). 

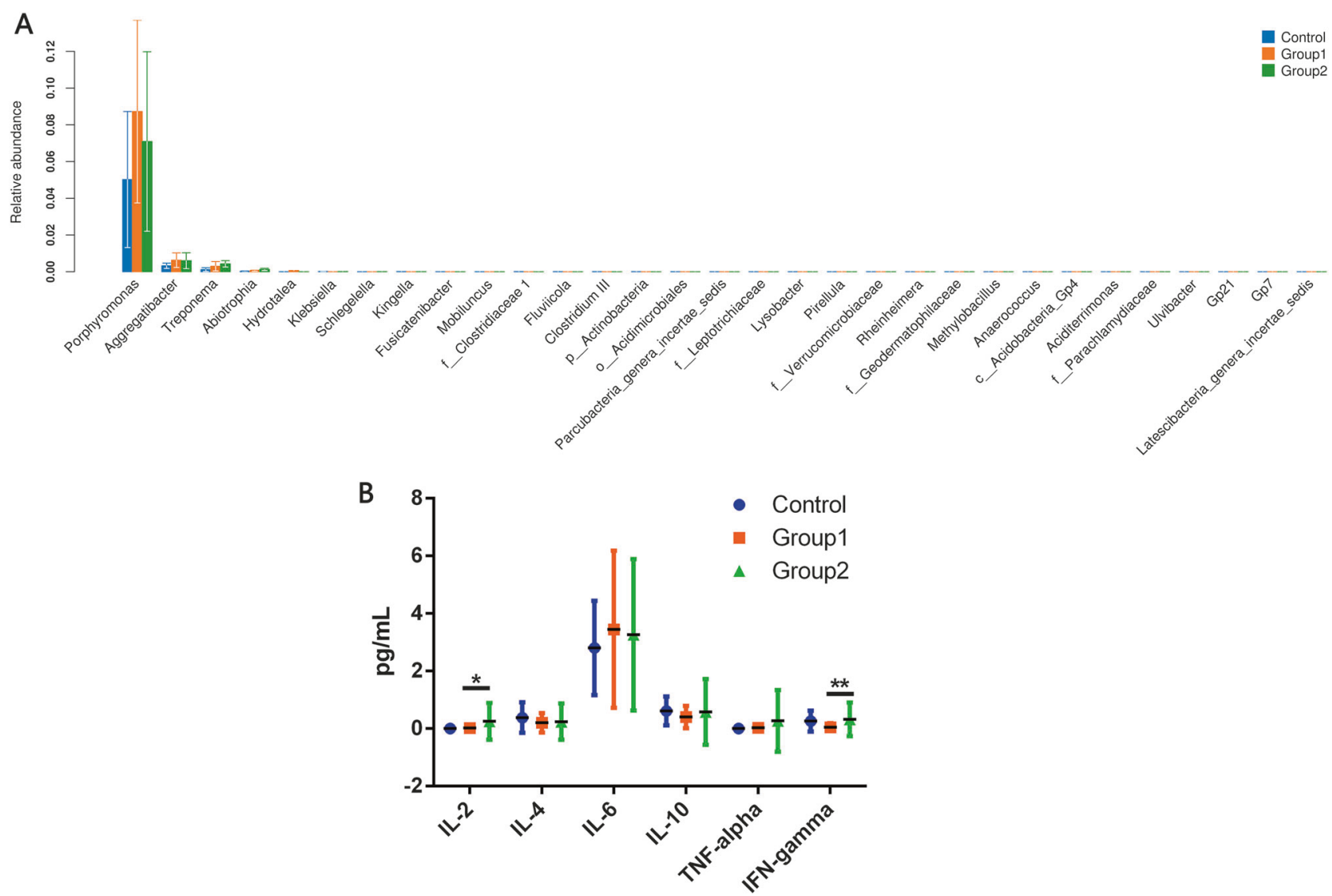

Fig. 1 Differences in the fecal microbiomes and cytokine levels in patients with obstructive sleep apnea-hypopnea syndrome (OSAHS) and the control group. There were significant differences in the fecal microbiome at the genera level (a), and the statistical analysis was performed with the Kruskal-Wallis test. Proinflammatory cytokines in patients with OSAHS (b); the statistical analysis was performed with

\section{Correlation between oral microbiota and homocysteine levels}

The correlations between HCY levels and the relative abundance of microbiota components are shown in Fig. 3; a correlation existed between HCY levels and the relative abundance of Aggregatibacter $(\mathrm{r}=-0.192, p=0.024)$ (see supplementary Table 5 for details).

\section{Discussion}

We conducted 16S rRNA pyrosequencing and bioinformatic analyses to compare the bacterial composition of oral samples from patients with OSAHS and HTN of varying severity. This approach enabled a relatively comprehensive description of the oral microbiome, with high levels of the Porphyromonas and Aggregatibacter genera being detected, accompanied by elevated levels of proinflammatory cytokines. Subsequently, data revealed that specific bacteria
Fisher's least significant difference test. $I L$ interleukin, TNF tumor necrosis factor, IFN interferon. Control: apnea-hypopnea index (AHI) $\leq 5$ (non-OSAHS), Group 1: $5<\mathrm{AHI} \leq 15$ (mild OSAHS with/without hypertension), Group 2: AHI $>15$ (moderate-to-severe OSAHS with/ without hypertension). $* P<0.05, * * P<0.01$

in the Porphyromonas genera were detected at high levels in patients with OSAHS with and without HTN and that the relative abundance of Aggregatibacter negatively correlated with the HCY levels. Moreover, the validated cutoff ROCAUC values of 0.759 for controls and patients with OSAHS and 0.641 for patients with OSAHS with and without HTN were detected. The functional analyses of the microbiome were also different, confirming that oral microbiota play a pathophysiological role in HTN in those patients.

OSAHS is an independent risk factor for CVDs, such as HTN [17, 22-24]. Most patients with OSAHS and comorbid HTN may develop refractory HTN, requiring a combination treatment with multiple antihypertensive medications, yet still achieving inadequate control of blood pressure [25]. The prevalence of HTN in the general population is $20 \%$; however, $\sim 50-60 \%$ of patients with OSAHS have comorbid HTN, and $~ 50 \%$ of patients with HTN have OSAHS [26]. It has been noted that patients with HTN have elevated oral bacterial counts [27, 28]. Studies have revealed that those who rarely brush their teeth are at a 


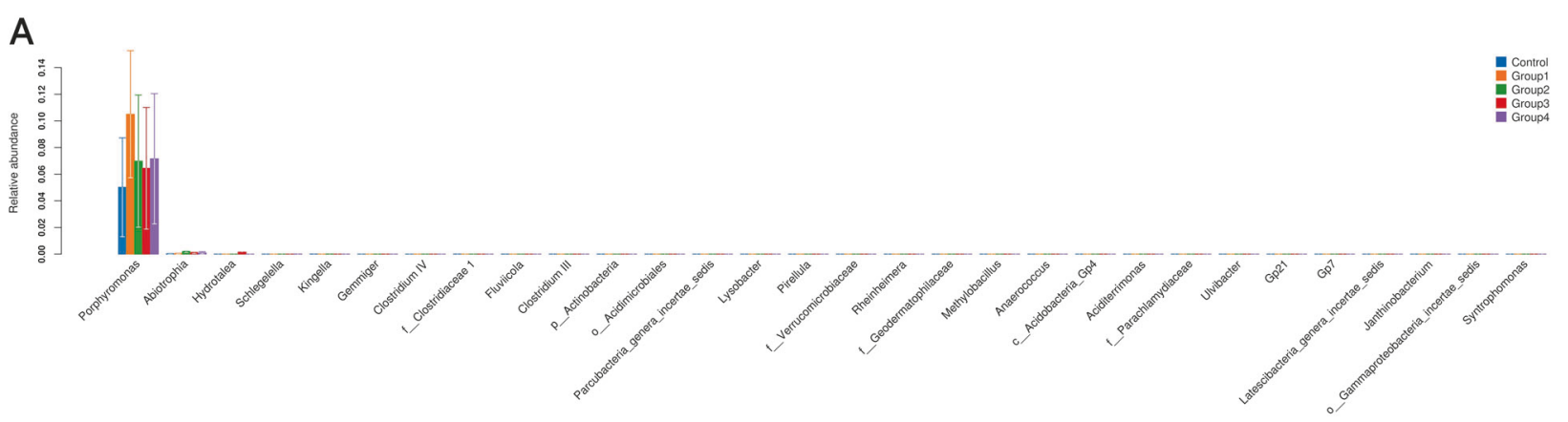

B

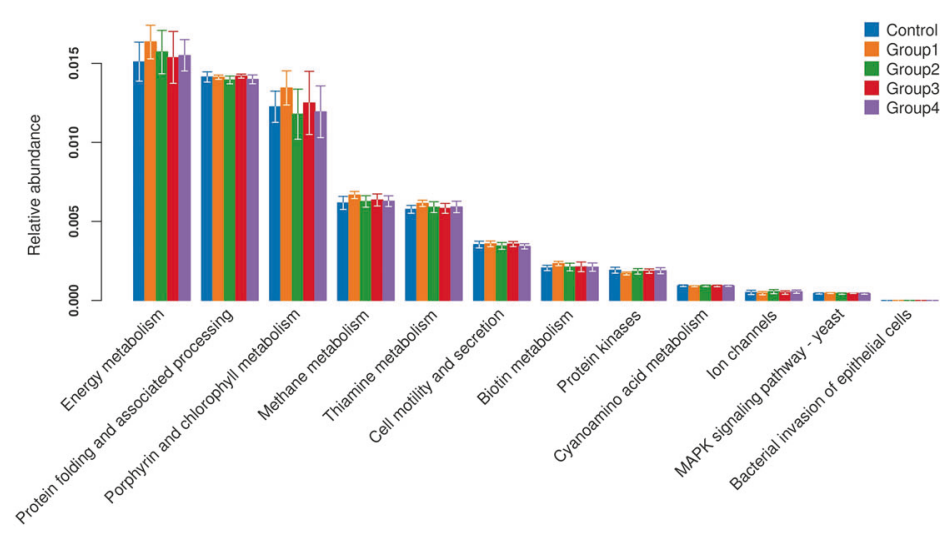

C

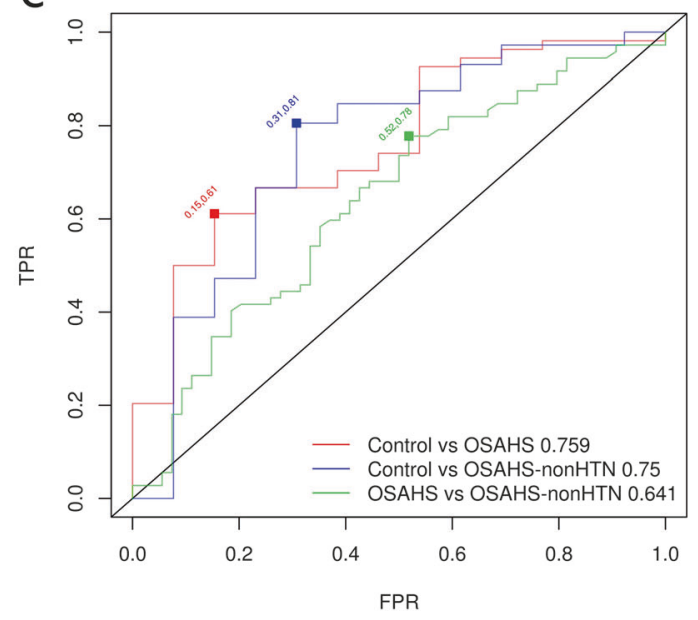

Fig. 2 Taxonomic differences in the fecal microbiota in the control group and patients with obstructive sleep apnea-hypopnea syndrome (OSAHS) with and without hypertension. There were significant differences in the fecal microbiome at the genera level (a). Kyoto Encyclopedia of Genes and Genomes pathways are shown for the fecal microbiome (b). Patients with OSAHS could be separated from controls with an area under the receiver operating characteristic curve (ROC-AUC) of 0.759; patients with OSAHS without HTN could be distinguished from the controls with an ROC-AUC of 0.750 ; and

significantly higher risk for HTN than those who brush after each meal; decreasing the frequency of brushing increases the incidence of HTN [27, 28].

The entry of oral microbiota and their metabolic products into systemic circulation is mechanism initiating cardiovascular events $[17,18]$. Aoyama et al[6]. compared the bacterial status in saliva and subgingival plaques in patients with periodontitis with and without HTN and found that those with HTN had a significantly higher detection rate of A. actinomycetemcomitans. Therefore, periodontopathogenic bacteria $(P$. gingivalis, Fusobacterium nucleatum, Prevotella intermedia, and Bacteroides forsythus) might enter atherosclerotic lesions directly and play a role in plaque formation [29].

In fact, periodontitis, an infectious disease of the toothsupporting structures caused by oral pathogens, has been found to be a risk factor for CVD [30]. Recently, it has been shown that patients with OSAHS have markedly higher oral bacterial counts than those of healthy individuals, and they patients with OSAHS could be distinguished from patients with OSAHS without HTN with an ROC-AUC of 0.641 (c). The statistical analysis was performed with the Kruskal-Wallis test. Control: apneahypopnea index (AHI) $\leq 5$ (non-OSAHS), Group 1: $5<\mathrm{AHI} \leq 15$ (mild OSAHS without hypertension), Group 2: AHI >15 (moderate-tosevere OSAHS without hypertension), Group 3: mild OSAHS with hypertension, Group 4: moderate-to-severe OSAHS with hypertension. FPR: false positive rate; TPR: true positive rate

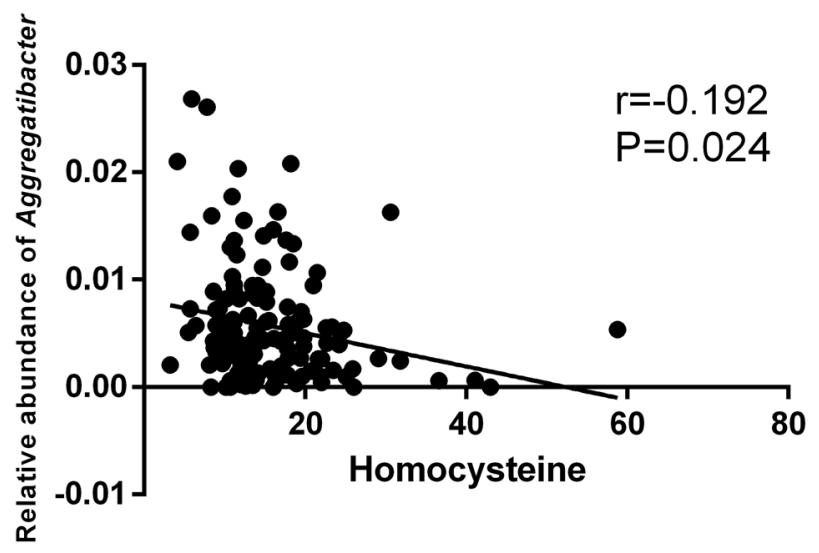

Fig. 3 The relative abundance of Aggregatibacter was negatively correlated with the homocysteine level

present with a relatively higher incidence of periodontitis $[15,31,32]$, which is consistent with our findings. We 
found that Aggregatibacter and Porphyromonas particularly had substantial effects.

However, the mechanism by which the oral microbiome contributes to CVD and HTN remains unclear. Direct invasion is an easy and fairly simple pathway. Evidence that identical oral bacterial pathogens are present in the subgingival and atherosclerotic plaques [29, 33] suggest that oral bacteria may directly invade atherosclerotic plaques, heart valves, and other cardiovascular tissues, as well as lesions, directly exerting their effects on CVDs. Based on our functional analyses of the microbiome, the bacterial invasion of the functional epithelial cells was significant in patients with OSAHS and HTN. Thus, we investigated whether the DNA of both $P$. gingivalis and A. actinomycetemcomitans existed in the blood to confirm the possible pathophysiological mechanisms involving the two organisms in OSAHS-related HTN.

Nevertheless, we found neither $P$. gingivalis nor A. actinomycetemcomitans genes in any of the patients. Pathogenic oral bacteria can induce proinflammatory factors [34] that mediate endothelial cell injury, increase vascular reactivity and resistance, inhibit vasodilators, and promote vasoconstrictor synthesis, vascular remodeling, and the occurrence and development of HTN [35]. We suggest that OSAHS-related HTN may result from relevant inflammatory markers in oral bacterial pathogen-induced pathways.

DNA and lipopolysaccharides in oral bacterial pathogens activate nuclear factor-kappa $\mathrm{B}(\mathrm{NF}-\mathrm{\kappa B})$ and activator protein 1 (AP1), resulting in the increased production of inflammatory factors, which synergize with bacterial cell wall components, activate neutrophils, and produce large amounts of reactive oxygen species (ROS) [16]. Additionally, hypoxia caused by OSAHS causes cell and tissue damage, which may lead to mitochondrial dysfunction, thereby affecting related enzyme systems, such as xanthine oxidase, endothelial nitric oxide (NO) synthase, nicotinamide adenine dinucleotide phosphate, and ROS production. These two pathways, when combined, produce excessive ROS and oxides, such as hydrogen peroxide and hydroxyl radicals, and the peroxides can react with NO to generate more free radicals. This process weakens the vasodilatory function of $\mathrm{NO}$ and severely compromises the function of endothelial cells. Furthermore, redox disequilibrium leads to excessive ROS, thereby aggravating the damage. In addition, studies have also shown that ROS participate in transduction in signaling pathways and promote NF- $\mathrm{\kappa B}$ or AP1 to produce excessive inflammatory factors, leading to increases in immune cells, monocytes, neutrophils, and lymphocytes, thereby contributing to the generation of more ROS, forming a vicious cycle $[36,37]$.

On the other hand, it has been noted that HCY levels are associated with an increased risk of metabolic abnormalities and HTN in patients with OSAHS [19]. However, our data revealed a negative correlation between Aggregatibacter and HCY levels. Theoretically, a positive correlation exists because A. actinomycetemcomitans contains the gene required for $\mathrm{HCY}$ synthesis and can thus stimulate HCY production; [38] this is also true for Porphyromonas [39]. However, the results of our functional analyses of the microbiome did not show the depletion of vitamin B6, B12, folates, or methionine in relation to HCY metabolism. Thus, future studies should reexamine these questions in subgroups of hypertensive and normotensive patients with OSAHS to assess the endothelial injury attributed to oral microbiome-produced HCY levels. Moreover, a comprehensive analysis of the possible mechanisms underlying oral microbiota-induced OSAHS-related HTN, including immune, inflammatory, and oxidative stress responses, should be performed.

\section{Conclusions}

Overall, we found evidence of changes in the oral microbiota in patients with OSAHS and an association between oral bacteria of the Porphyromonas and Aggregatibacter genera and OSAHS-linked HTN. Our findings regarding the close association of some oral microbiota with HTN and the clinical characteristics of CVD may also enhance our understanding of the pathogenesis of OSAHS-linked HTN by elucidating the basis for the differences in the oral microbiome. Moreover, this could support effective intervention for, management of, and prognostic evaluations of modifying oral microbiota in patients with OSAHS.

Acknowledgements We thank all the patients and their families who participated in this study. The authors appreciate the assistance provided by Huan Wu (G-BIO Biotech, Inc., Hangzhou, China), who performing the bioinformatic analysis. The authors would also like to thank the Fujian Provincial Health and Family Planning Commission, China, under contract No. 2018-CX-36; the Fujian Province Science and Technology Project, China, under contract No. 2018J01290 and No. 2015J01445; and the Quanzhou Science and Technology Project, China, under contract No. 2018Z107, No. 2017Z016, and No.2013Z55; and contract No. 2014JQNJC20 for the Young People Training Project from the Fujian Province Health System. This article was also subsidized by the Strait Exchanges Postdoctoral Funding Schemes of Fujian Province, China, and academic funding from the Second Affiliated Hospital of Fujian Medical University (serial No. BSH001).

Author contributions Conception and design: CYK, YMZ. Acquisition of data: HPZ, CYK, AKH, LMH, HZS, FRY, XBZ. Analysis and interpretation of data: CYK, DC, HPZ, AKH, YMZ. Drafting or revision of the article: $\mathrm{CYK}, \mathrm{DC}, \mathrm{AKH}, \mathrm{YMZ}$.

\section{Compliance with ethical standards}

Conflict of interest The authors declare that they have no conflict of interest. 
Publisher's note: Springer Nature remains neutral with regard to jurisdictional claims in published maps and institutional affiliations.

Open Access This article is licensed under a Creative Commons Attribution 4.0 International License, which permits use, sharing, adaptation, distribution and reproduction in any medium or format, as long as you give appropriate credit to the original author(s) and the source, provide a link to the Creative Commons license, and indicate if changes were made. The images or other third party material in this article are included in the article's Creative Commons license, unless indicated otherwise in a credit line to the material. If material is not included in the article's Creative Commons license and your intended use is not permitted by statutory regulation or exceeds the permitted use, you will need to obtain permission directly from the copyright holder. To view a copy of this license, visit http://creativecommons. org/licenses/by/4.0/.

\section{References}

1. Chami HA, Resnick HE, Quan SF, Gottlieb DJ. Association of incident cardiovascular disease with progression of sleepdisordered breathing. Circulation. 2011;123:1280-6.

2. Benjamin EJ, Blaha MJ, Chiuve SE, Cushman M, Das SR, Deo R, American Heart Association Statistics Committee and Stroke Statistics Subcommittee. et al. Heart disease and stroke statistics2017 update: a report from the American Heart Association. Circulation. 2017;135:146-603.

3. Parati G, Lombardi C, Narkiewicz K. Sleep apnea: epidemiology, pathophysiology, and relation to cardiovascular risk. Am J Physiol Regul Integr Comp Physiol. 2007;293:1671-83.

4. Pedrosa RP, Drager LF, Gonzaga CC, Sousa MG, de Paula LK, Amaro AC, et al. Obstructive sleep apnea: the most common secondary cause of hypertension associated with resistant hypertension. Hypertension. 2011;58:811-7.

5. He QY, Feng J, Zhang XL, Liang ZA, Huang SG, Kang J, Sleep Breath Disorder Group; Society of Respiratory Medicine; Chinese Medical Association. et al. Relationship of daytime blood pressure and severity of obstructive sleep apnea among Chinese: a multicenter investigation in China. Chin Med J. 2010;123:18-22.

6. Aoyama N, Suzuki JI, Kumagai H, Ikeda Y, Akazawa H, Komuro I, et al. Specific periodontopathic bacterial infection affects hypertension in male cardiovascular disease patients. Heart Vessels. 2018:33:198-204.

7. Durgan DJ, Ganesh BP, Cope JL, Ajami NJ, Phillips SC, Petrosino JF, et al. Role of the gut microbiome in obstructive sleep apnea-induced hypertension. Hypertension. 2016;67:469-74.

8. Ueno M, Izumi Y, Kawaguchi Y, Ikeda A, Iso H, Inoue M, Tsugane S, JPHC Study Group. Prediagnostic plasma antibody levels to periodontopathic bacteria and risk of coronary heart disease. Int Heart J. 2012;53:209-14.

9. Yang T, Santisteban MM, Rodriguez V, Li E, Ahmari N, Carvajal $\mathrm{JM}$, et al. Gut dysbiosis is linked to hypertension. Hypertension. 2015;65:1331-40.

10. Zadik Y, Bechor R, Galor S, Justo D, Heruti RJ. Erectile dysfunction might be associated with chronic periodontal disease: two ends of the cardiovascular spectrum. J Sex Med. 2009;6:1111-6.

11. Robles-Vera I, Toral M, Romero M, Jiménez R, Sánchez M, Pérez-Vizcaíno F, et al. Antihypertensive effects of probiotics. Curr Hypertens Rep. 2017;19:26.

12. Schippa S, Conte MP. Dysbiotic events in gut microbiota: impact on human health. Nutrients. 2014;6:5786-805.

13. Farré N, Farré R, Gozal D. Sleep apnea morbidity: a consequence of microbial-immune cross-talk? Chest. 2018;154:754-9.

14. Kato Y, Hagiwara M, Ishihara Y, Isoda R, Sugiura S, Komatsu T, et al. TNF- $\alpha$ augmented porphyromonas gingivalis invasion in human gingival epithelial cells through Rab5 and ICAM-1. BMC Microbiol. 2014;14:229.

15. Nizam N, Basoglu OK, Tasbakan MS, Lappin DF, Buduneli N. Is there an association between obstructive sleep apnea syndrome and periodontal inflammation? Clin Oral Investig. 2016;20: 659-68.

16. Dahiya P, Kamal R, Gupta R, Bhardwaj R, Chaudhary K, Kaur S. Reactive oxygen species in periodontitis. J Indian Soc Periodontol. 2013;17:411-6.

17. Klarström Engström $\mathrm{K}$, Khalaf $\mathrm{H}$, Kälvegren $\mathrm{H}$, Bengtsson $\mathrm{T}$. The role of Porphyromonas gingivalis gingipains in platelet activation and innate immune modulation. Mol Oral Microbiol. 2015;30:62-73.

18. Chukkapalli SS, Easwaran M, Rivera-Kweh MF, Velsko IM, Ambadapadi S, Dai J, et al. Sequential colonization of periodontal pathogens in induction of periodontal disease and atherosclerosis in LDLR null mice. Pathog Dis. 2017;75:1-10.

19. Monneret D, Tamisier R, Ducros V, Garrel C, Levy P, Baguet JP, et al. The impact of obstructive sleep apnea on homocysteine and carotid remodeling in metabolic syndrome. Respir Physiol Neurobiol. 2012;180:298-304.

20. Kim J, Lee SK, Yoon DW, Shin C. Concurrent presence of obstructive sleep apnea and elevated homocysteine levels exacerbate the development of hypertension: A KoGES six-year follow-up study. Sci Rep. 2018;8:2665.

21. Kao YC, Ko CY, Wang SC, Liu YP. Protective effects of quetiapine on metabolic and iflammatory abnormalities in schizophrenic patients during exacerbated stage. Chin J Physiol. 2016;59:69-77.

22. Nieto FJ, Young TB, Lind BK, Shahar E, Samet JM, Redline S, et al. Association of sleep-disordered breathing, sleep apnea, and hypertension in a large community-based study. Sleep Heart Health Study. JAMA. 2000;283:1829-36.

23. Shahar E, Whitney CW, Redline S, Lee ET, Newman AB, Nieto FJ, et al. Sleep-disordered breathing and cardiovascular disease: cross-sectional results of the Sleep Heart Health Study. Am J Respir Crit Care Med. 2001;163:19-25.

24. Shamsuzzaman AS, Gersh BJ, Somers VK. Obstructive sleep apnea: implications for cardiac and vascular disease. JAMA. 2003;290:1906-14.

25. Logan AG, Perlikowski SM, Mente A, Tisler A, Tkacova R, Niroumand $M$, et al. High prevalence of unrecognized sleep apnoea in drug-resistant hypertension. J Hypertens. 2001;19: 2271-7.

26. Goodfriend TL, Calhoun DA. Resistant hypertension, obesity, sleep apnea, and aldosterone: theory and therapy. Hypertension. 2004;43:518-24.

27. Fujita M, Ueno K, Hata A. Lower frequency of daily teeth brushing is related to high prevalence of cardiovascular risk factors. Exp Biol Med. 2009;234:387-94.

28. de Oliveira C, Watt R, Hamer M. Toothbrushing, inflammation, and risk of cardiovascular disease: results from Scottish Health Survey. BMJ. 2010;340:2451.

29. Zhong LJ, Zhang YM, Liu H, Liang P, Murat AR, Askar $S$. Detection of periodontal pathogens in coronary atherosclerotic plaques. Zhonghua Kou Qiang Yi Xue Za Zhi. 2008;43:4-7.

30. Destefano F, Anda RF, Kahn HS, Williamson DF, Russell CM. Dental disease and risk of coronary heart disease and mortality. BMJ. 1993;306:688-91.

31. Gunaratnam K, Taylor B, Curtis B, Lappin DF, Buduneli N. Obstructive sleep apnoea and periodontitis: a novel association? Sleep Breath. 2009;13:233-9.

32. Seo WH, Cho ER, Thomas RJ, An SY, Ryu JJ, Kim H, et al. The association between periodontitis and obstructive sleep apnea: a preliminary study. J Periodontal Res. 2013; 48:500-6. 
33. Oliveira FA, Forte CP, Silva PG, Lopes CB, Montenegro RC, Santos ÂK, et al. Molecular analysis of oral bacteria in heart valve of patients with cardiovascular disease by real-time polymerase chain reaction. Medicine. 2015;94:2067.

34. Chandy S, Joseph K, Sankaranarayanan A, Issac A, Babu G, Wilson B, et al. Evaluation of C-reactive protein and fibrinogen in patients with chronic and aggressive periodontitis: a clinical biochemical study. J Clin Diagn Res. 2017;11:41-5.

35. Wu CX, Liu Y, Zhang JC. Chronic intermittent hypoxia and hypertension: a review of systemic inflammation and Chinese medicine. Chin J Integr Med. 2013;19:394-400.
36. Lavie L, Lavie P. 3Molecular mechanisms of cardiovascular disease in OSAHS: the oxidative stress link. Eur Respir J. 2009;3:1467-84

37. Lavie L. Oxidative stress in obstructive sleep apnea and intermittent hypoxia-revisited-the bad ugly and good: implications to the heart and brain. Sleep Med Rev. 2015;20:27-45.

38. Shao H, Lamont RJ, Demuth DR. Autoinducer 2 is required for biofilm growth of Aggregatibacter (Actinobacillus) actinomycetemcomitans. Infect Immun. 2007;75:4211-8.

39. Yoshida A, Yoshimura M, Ohara N, Yoshimura S, Nagashima S, Takehara T, et al. Hydrogen sulfide production from cysteine and homocysteine by periodontal and oral bacteria. J Periodontol. 2009;80:1845-51.

\section{Affiliations}

\section{Chih-Yuan $\mathrm{Ko}^{1,2,3,4} \cdot$ An-Ke Hu$u^{1,2,3} \cdot$ Dylan Chou ${ }^{5} \cdot$ Li-Mei Huang ${ }^{1,2,3} \cdot$ Huan-Zhang Su ${ }^{1,2,3} \cdot$ Fu-Rong Yan ${ }^{1,2,3,6}$. Xiao-Bin Zhang ${ }^{1,2,3} \cdot$ Hua-Ping Zhang ${ }^{1,2,3} \cdot$ Yi-Ming Zeng ${ }^{1,2,3}$}

1 Department of Respiratory and Critical Care Medicine, the Second Affiliated Hospital of Fujian Medical University, 362000 Quanzhou, China

2 Respiratory Medicine Center of Fujian Province, 362000 Quanzhou, China

3 Key Laboratory of Fujian Medical University, Fujian Province University, 362000 Quanzhou, China

4 Department of Endocrinology and Metabolism, the Second
Affiliated Hospital of Fujian Medical University, 362000 Quanzhou, China

5 Zhuhai Campus of Zunyi Medical University, Zhuhai, 519090 Guangdong, China

6 Center for Molecular Diagnosis and Therapy, the Second Affiliated Hospital of Fujian Medical University, 362000 Quanzhou, China 